\title{
SPASMOPHILIA AND THE ALKALI RESERVE OF THE BLOOD*
}

\author{
JOSEPH K. CALVIN, S.B., M.D. \\ AND \\ MAXWELL P. BOROVSKY, S.B., M.D. \\ CHICAGO
}

Wilson and his co-workers ${ }^{1}$ pointed out that in parathyroidectomized dogs, just previous to the attacks of tetany, an increased alkalinity of the blood develops, a condition termed by them "alkalosis," which is neutralized by the acid products generated by the tetanic contractions of the musculature. Recently Grant and Goldman ${ }^{2}$ have shown that forced respiration may cause symptoms of tetany to appear in the human. In these cases the underlying factor appears to be an "alkalosis." The forced breathing causes an excess of carbon dioxid to be exhaled from the lungs, thereby reducing the amount in the blood. This results in an increased alkalinity of the blood. However, they observed that the tetanic movements apparently produce acid products which tend to compensate for the increased alkalinity until a readjustment takes place.

Clinically it has at times been noted ${ }^{3}$ that after the therapeutic administration of sodium bicarbonate for acidosis, characteristic symptoms of tetany may develop. These symptoms cease promptly when the sodium bicarbonate is stopped.

Howland and Marriott ${ }^{3}$ have made determinations of the hydrogen ion concentration of the serum of infants with tetany by the dialysis indicator method, but have not found an "alkalosis."

Acute infections frequently precipitate active manifestations of spasmophilia in those having the latent form previous to the infections. As acute infections are usually accompanied by a tendency to acidosis, alkalosis could scarcely be a factor in producing tetany in this type of case.

Jones and $\mathrm{Nye}^{4}$ found no relation between the alkali reserve and the concentration of calcium and phosphoric acid in the blood. Other investigators believe that the hydrogen ion concentration of

* Received for publication, Feb. 25, 1922.

* From the Department of Pediatrics, University of Illinois, and Children's Department, Cook County Hospital.

1. Wilson, Stearns and Thurlow: J. Biol. Chem. 23:89, 1915. Wilson, Stearns and Janney: ibid. 23:123, 1915.

2. Grant, S. B., and Goldman, A.: Am. J. Physiol. 52:209, 1920.

3. Howland, J., and Marriott, W. McK.: Quart. J. Med. 11:289, 1917.

4. Jones, M. R., and Nye, L. I.: J. Biol. Chem. 47:331, 1921. 
the serum (and the factors regulating this as the carbon dioxid tension and the alkali reserve) influence the type of calcium and phosphate salts formed, and consequently the relative amounts of calcium and phosphate ions available and the solubility of their salts. ${ }^{5}$

We determined the carbon dioxid combining power of the blood plasma by the Van Slyke $\operatorname{method}^{6}$ in several cases of active spasmophilia in infants and again after their recovery.

\section{REPORT OF CASES}

The following are the histories, in abstract, of the patients we have studied:

CASE 1.-G. D., aged 15 months, colored, weight 14 pounds, was admitted to Cook County Children's Hospital, April 3 with evidence of an early bronchopneumonia and signs of active tetany consisting of marked laryngospasm, Chvostek, Trousseau and an Erb's electrical reaction of C.O.C. $=3.5 \mathrm{ma}$. There were also signs of marked rickets. The tetany cleared up on treatment with calcium bromid, and cod liver oil with phosphorus, and was reprecipitated by a course of sodium chlorid, 15 grains, and potassium citrate, 10 grains, given once daily. Six days after starting the sodium and potassium salts there was a definite Chvostek, a moderate Trousseau and a C.O.C. of 2.5 ma. Blood was drawn at this time from the longitudinal sinus to be examined by the Van Slyke method for a determination of the carbon dioxid combining power, and it was found that there were 40 c.c. of carbon dioxid bound as bicarbonate by 100 c.c. of plasma. The patient was again placed on calcium lactate, 3 grains, twice daily, and cod liver oil with phosphorus, 1 dram, three times daily, after stopping the sodium and potassium salts. At the end of six days of this treatment, the C.O.C. was 15 ma., there was no carpopedal spasm and only a very faint Chvostek. The blood reading showed 53.3 c.c. of carbon dioxid bound as bicarbonate by 100 c.c. of plasma. Eighteen days later there were still no clinical manifestations of spasmophilia and the blood reading was 46.2 c.c. of carbon dioxid.

CASE 2.-E. M., aged 3 years, colored, weight 17 pounds, was admitted May 23. 1921, suffering from pertussis, very marked rickets and active tetany consisting of marked laryngospasm, Chvostek and C.O.C. of 4 ma. with generalized convulsions. The next day the child was placed on 1 dram of phosphorized cod liver oil and calcium lactate, 5 grains, three times daily, and antispasmodics for the pertussis. The day that this treatment was instituted the blood reading was 42.4 c.c. of carbon dioxid, the spasmophilic signs were the same, although no convulsion had occurred during the previous eighteen hours. The C.O.C. was 3.5 ma. On examination twenty-two days later there were no signs of spasmophilia; C.O.C. $12 \mathrm{ma}$., and the blood ${ }^{7}$ reading was 59 c.c. of carbon dioxid. Nine days later there were still no clinical manifestations of tetany, and the blood reading showed 55.3 c.c. of carbon dioxid combined as bicarbonate to 100 c.c. of plasma.

CASE 3.-M. M., twin of E. M., was admitted the same day and with the same clinical findings but no generalized convulsions. C.O.C. $=4 \mathrm{ma}$. The blood reading on admission was 47 c.c. of carbon dioxid. After having been on cod liver oil and calcium lactate for eleven days with no signs of tetany, the blood ${ }^{7}$ reading was 50 c.c. of carbon dioxid.

5. Howland, J., and Kramer, B.: Am. J. Dis. Child. 22:105 (July) 1921.

6. Van Slyke, D. D., and Cullen, G. E.: J. Biol. Chem. 30:317, 1917.

7. The blood was obtained from the external jugular vein. 


\section{CONCLUSIONS}

In the cases of spasmophilia in the infants studied, no evidence of "alkalosis" was found. In fact, there was a tendency for the alkali reserve to be lowered during the spasmophilic stage and gradually to be increased as the cases recovered.

It appears that the symptoms of tetany may be produced in a variety of ways, but the evidence is not forthcoming that "alkalosis" plays a part in the genesis of infantile spasmophilia.

We wish to thank Dr. Welker and the Department of Physiological Chemistry of the University of Illinois for the privilege of using their laboratories for the blood chemistry work. 